\title{
Gallium nitride MEMS resonators: how residual stress impacts design and performances
}

Christophe Morelle1, Didier Théron ${ }^{1}$, Joff Derluyn ${ }^{2}$, Stefan Degroote ${ }^{2}$, Marianne Germain ${ }^{2}$, Victor Zhang ${ }^{1}$, Lionel Buchaillot ${ }^{1}$, Bertrand Grimbert ${ }^{1}$, Pascal Tilmant ${ }^{1}$, François Vaurette ${ }^{1}$, Isabelle Roch-Jeune ${ }^{1}$, Virginie Brandli ${ }^{1}$, Vanessa Avramovic ${ }^{1}$, Etienne Okada $^{1}$, and Marc Faucher ${ }^{1}$.

\author{
${ }^{1}$ IEMN, UMR CNRS 8520 \\ Avenue Poincaré CS 60069, 59652 Villeneuve d'Ascq Cedex, France \\ christophe.morelle@isen.iemn.univ-lille1.fr; marc.faucher@isen.iemn.univ-lille1.fr \\ ${ }^{2}$ EpiGaN nv, Kempische steenweg 293, 3500, Hasselt, Belgium
}

\begin{abstract}
Starting from Gallium Nitride (GaN) epitaxially grown on silicon, pre-stressed micro-resonators with integrated piezoelectric transducers have been designed, fabricated, and characterized. In clamped-clamped beams, it is well known that tensile stress can be used to increase the resonant frequency. Here we calculate the mode shape functions of out-of-plane flexural modes in prestressed beams and we derive a model to predict both the resonant frequency and the piezoelectric actuation factor. We show that a good agreement between theory and experimental results can be obtained and we derive the optimal design for the electromechanical transduction. Finally, our model predicts an increase of the quality factor due to the tensile stress, which is confirmed by experimental measurements under vacuum. This study demonstrates how to take advantage from the material quality and initial stress resulting of the epitaxial process.
\end{abstract}

Acknowledgment This work has been supported by the French National Agency (ANR) with the project ASTRID AMGASI ANR-11-ASTR0037. We thank the Direction Générale de l'Armement (DGA) and Région Nord-Pas-de-Calais for financial support. This work was partly supported by the French RENATECH network.

\section{Introduction}

Gallium nitride $(\mathrm{GaN})$ is the second most used material in microelectronics after silicon, due to its success in optoelectronics (DenBaars et al. 2013), and in high power electronics and RF (Khan et al. 1993; Mishra et al. 2008). Its piezoelectric and mechanical properties make it also a promising microelectromechanical systems (MEMS) material for sensors. The combination of piezoelectricity, mechanical characteristics, chemical inertness and high breakdown voltage can lead to sensors that overcome some limitations of silicon (Cimalla et al. 2007). Moreover, the use of AlGaN/GaN heterostructures enables efficient transduction and integration of electronics in the same package (Ansari et al. 2011; Popa and Weinstein 2013).

Previously, we investigated beam resonators based on GaN with piezoelectric actuator and integrated HEMT transducers (Faucher et al. 2012; Faucher et al. 2009). The material grown by MOCVD presented a reproducible high tensile stress due to the epitaxial growth process. In this work, we study the effect of the tensile stress on the resonant frequencies and the flexural modes of vibration. It leads to modification in the design of the transducers for optimal performances. Moreover, the tensile stress induces a higher potential energy stored by the vibrating beam which increases the quality factor.

\section{Derivation of frequency and mode shape for arbitrary mode with stress}

Performances of resonant sensors are linked to their resonant frequencies. For beams vibrating with flexion modes, assuming small amplitudes compared to the dimensions of the beam, and elastic deformation, the resonant frequencies and the associated motion can be calculated from the Euler-Bernoulli equation (Brueckner et al. 2007). Its expression is given in Fig. 1a. For calculating the resonant frequencies of doubly clamped beams, we cancel the driving force and the damping coefficient and assume sinusoidal motion. This leads to a characteristic equation (Torri et al. 2014) that we solve numerically. The solution is plotted in Fig. $1 \mathrm{~b}$ using dimensionless quantities representing frequency and stress. Therefore, it can be used for any kind of doubly clamped beam: this solution is independent of the dimensions and the material properties of the beam. 
(a) $E_{e f} I \frac{\partial^{4} U(x, t)}{\partial x^{4}}-\sigma A \frac{\partial^{2} U(x, t)}{\partial x^{2}}+\rho A \frac{\partial^{2} U(x, t)}{\partial t^{2}}+\alpha \frac{\partial U(x, t)}{\partial t}=p(x, t)$

(b)

$U(x, t)$ : vertical displacement of the beam along its length $E_{\text {ef }}$ : effective Young's modulus

$I$ : second moment of area along the longitudinal axis

$\sigma$ : stress (positive for tension, negative for compression)

$A$ : cross-section area of the beam

$\rho$ : mass density of the beam

$\alpha$ : damping coefficient per unit length

$p$ : driving force per unit length

$f$ : resonant frequency

$L$ : beam length

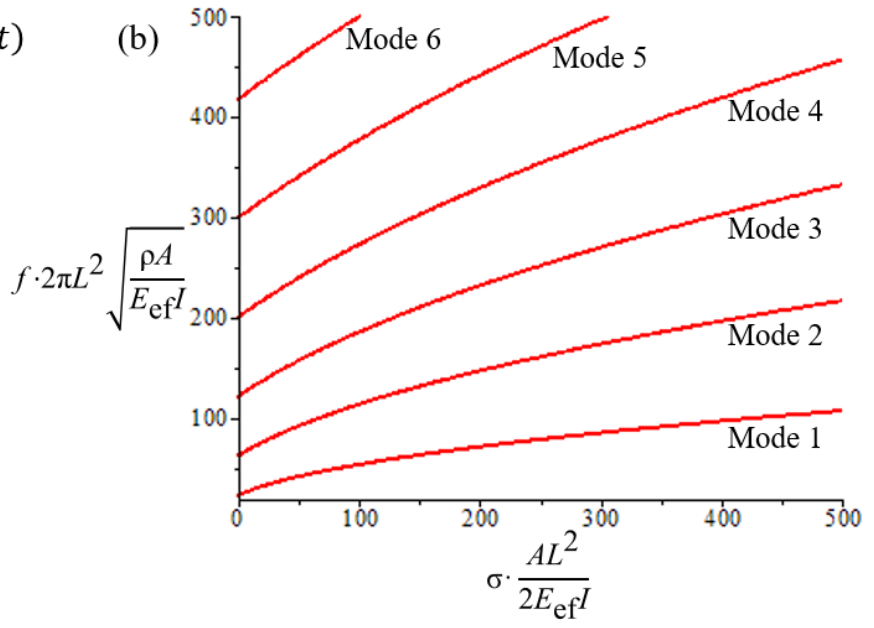

Fig. 1 a Euler-Bernoulli equation with uniform axial stress. b Resonant frequencies of doubly clamped beam for varying tensile stress, expressed with dimensionless quantities

Thanks to the Euler-Bernoulli equation (Fig. 1a), the mode shape functions can be obtained for any mode at any tensile stress. The Fig. 2 gives the mode shape function of the first mode normalized to a maximum amplitude equal to 1 for different tensile stresses, and its first derivative. Obviously, the case of infinite tensile stress cannot occur in reality because the beam would fracture, but it also represents the case of very thin and long beams reaching the behavior of a vibrating string.

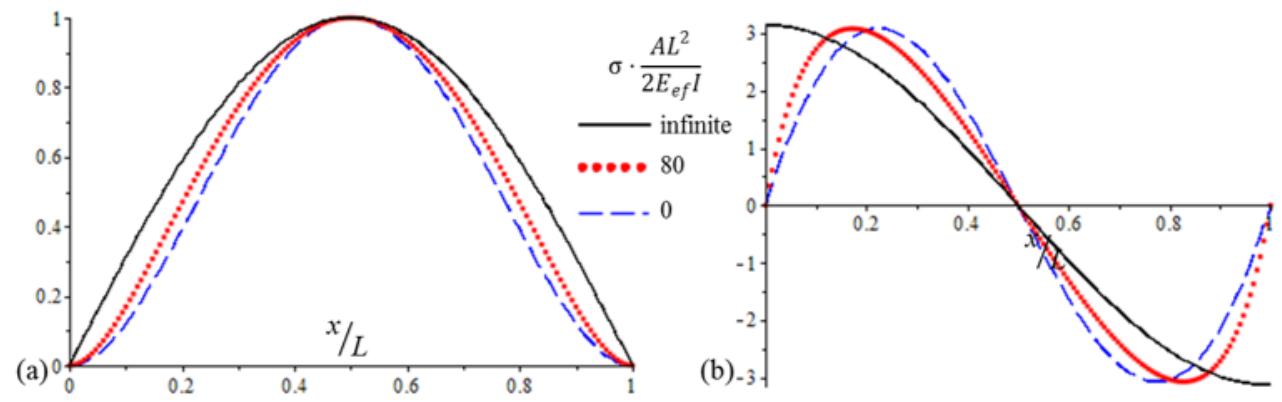

Fig. 2 Normalized mode shape function of the first mode (a) and its first derivative (b) for various tensile stress. The blue dashed line is the mode shape function without tensile stress, and the black solid line is the mathematical limit of infinite tensile stress (or vibrating string limit)

The resonant frequencies can be expressed analytically using the Rayleigh method, based on energy conservation. At a resonant frequency $f_{n}$, the vertical displacement of the beam can be written as $U(x, t)=B \cdot U_{n}(x) \cdot \cos \left(2 \pi f_{n} t+\varphi\right)$, where $U_{n}(x)$ is the mode shape function that is defined up to a multiplicative constant, and $B$ is a constant that depends on the definition used for the mode shape function and the driving force. Due to the axial stress, a potential energy is added to the system. Here are the expressions of the kinetic energy $E_{c}$, the flexural potential energy $E_{p, f l e x}$ and the axial potential energy $E_{p, \text { axial }}$ :

$E_{c}=B^{2} \frac{\rho A}{2}\left(2 \pi f_{n}\right)^{2} \int_{0}^{L} U_{n}^{2} d x$

(1)

$E_{p, \text { flex }}=B^{2} \frac{E_{e f} I}{2} \int_{0}^{L}\left(\frac{\partial^{2} U_{n}}{\partial x^{2}}\right)^{2} d x$

(2)

$E_{\text {p,axial }}=B^{2} \frac{\sigma A}{2} \int_{0}^{L}\left(\frac{\partial U_{n}}{\partial x}\right)^{2} d x$

(3) 
With these definitions of the potential energies, the conservation of the energy during the motion of the beam corresponds to $E_{c}=E_{p, \text { flex }}+E_{p, a x i a l}$. This leads to the equation of the frequency of the $n^{\mathrm{th}}$ mode, which also corresponds exactly to the numerical solution given by Fig. 1b:

$f_{n}=\frac{a_{n}}{2 \pi L^{2}} \sqrt{\frac{E_{e f} I}{\rho A}} \sqrt{1+\frac{\gamma_{n} \sigma A L^{2}}{12 E_{e f} I}}=\frac{k_{n}{ }^{2}}{2 \pi L^{2}} \sqrt{\frac{E_{e f} I}{\rho A}} \sqrt{1+\frac{\gamma_{n}^{\prime} \sigma A L^{2}}{12 E_{e f} I}}$

Here $\quad a_{n}=L^{2}\left[\left(\int_{0}^{L}\left(\frac{\partial^{2} U_{n}(x)}{\partial x^{2}}\right)^{2} d x\right) /\left(\int_{0}^{L} U_{n}^{2}(x) d x\right)\right]^{1 / 2}$ and $\quad \gamma_{n}=\left(12 \int_{0}^{L}\left(\frac{\partial U_{n}(x)}{\partial x}\right)^{2} d x\right) /\left(L^{2} \int_{0}^{L}\left(\frac{\partial^{2} U_{n}(x)}{\partial x^{2}}\right)^{2} d x\right) \quad$ are dimensionless coefficients, independent of the beam dimensions for a fixed mode shape function. The $a_{n}$ coefficients are linked to the ratio between the flexural potential energy and the kinetic energy, and the $\gamma_{n}$ coefficients are linked to the ratio between the axial potential energy and the flexural potential energy. Without stress, $a_{n}=k_{n}{ }^{2}$ where $k_{n}$ are the well-known eigenvalues 4.730 , $7.853, \ldots,(\mathrm{n}+1 / 2) \pi ;$ and $\gamma_{n} \approx 0.2949,0.1453, \ldots, 12\left(k_{n}-2\right) / k_{n}{ }^{3}$. Since the mode shape functions vary with the stress, the coefficients $a_{n}$ and $\gamma_{n}$ are modified by the stress: for increasing stress, $a_{n}$ increases up to infinite whereas $\gamma_{n}$ decreases toward zero.

However, the variation of $a_{n}$ and $\gamma_{n}$ can be taken into account using a unique coefficient: $a_{n}$ is replaced by the fixed $k_{n}{ }^{2}$ and $\gamma_{n}$ is replaced by $\gamma_{n}^{\prime}$, which leads to the second form of equation (4). Without stress the new coefficient $\gamma^{\prime}{ }_{n}$ is equal to $\gamma_{n}$ and for increasing tensile stress $\gamma_{n}{ }_{n}$ decreases toward $12 n^{2} \pi^{2} / k_{n}{ }^{4}$. It gives a limit of about 0.2366 and 0.1246 for the first and second mode respectively. A unique coefficient that does not diverge is easier to use, and then it is often employed to calculate the resonant frequency of doubly clamped beams with internal stress. With this unique coefficient, the resonant frequency with or without tensile stress can be easily compared. However, the two coefficients $a_{n}$ and $\gamma_{n}$ can be directly expressed using the different contributions of the energy of the system, which is not the case of $\gamma^{\prime}{ }_{n}$.

\section{Vibration amplitude and experimental measurements}

We fabricated doubly clamped beams in GaN from EpiGaN GaN-on-Si substrate with dimensions of $400 \mu \mathrm{m} \times 40 \mu \mathrm{m} \times 1.8 \mu \mathrm{m}$ (Fig. 3). Details about the fabrication process can be found elsewhere (Faucher et al. 2012). The actuation is provided by a Schottky diode with a length of $50 \mu \mathrm{m}$ and by using the two-dimensional electron gas (2-DEG) as a bottom electrode to apply the high electric field on the thin AlGaN layer. The detection is performed by a resonant high-electron-mobility transistor (R-HEMT) integrated on the beam. When the beam vibrates, the strain of the GaN material generates piezoelectric charge in the 2-DEG. This charge is converted into current and amplified by the transistor. From the measured resonant frequencies of the different modes, we deduce the stress of the beam using the previous model. The value of $\sigma A L^{2} /\left(2 E_{\mathrm{e} f} I\right)$ is found to be about 80 . It corresponds to a tensile stress of about $100 \mathrm{MPa}$, which is in good agreement with the value expected from the epitaxial growth process. This value is found assuming that the effective Young's modulus of the beam is about $290 \mathrm{GPa}$ which fits with the resonant frequencies of the modes 1 to 9. The value of the Young's modulus has also been confirmed by nanoindentation and is coherent with values previously reported for similar structures (Leclaire et al. 2014). The growth process conciliates the high tensile stress with the Young's modulus among the highest reported for thin GaN (Cimalla et al. 2007). The mode shape function of the first mode calculated for this stress value is reported in Fig. 2.

(a)

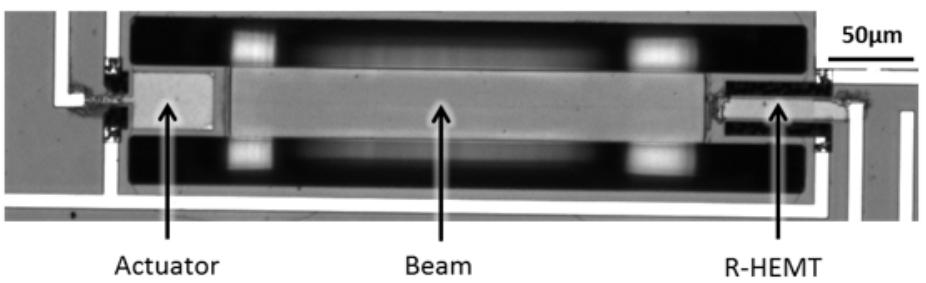

(b)

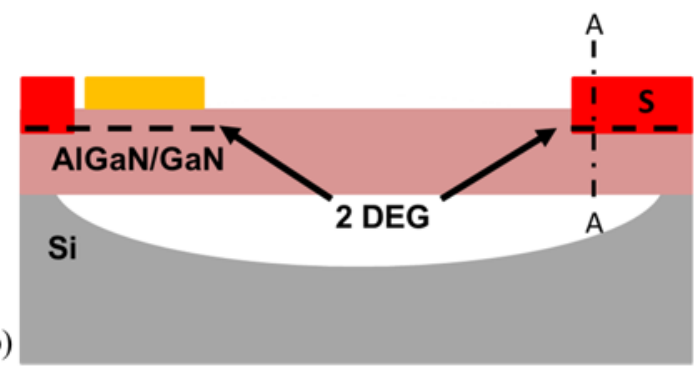

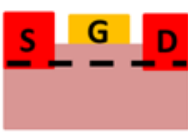

Section A-A

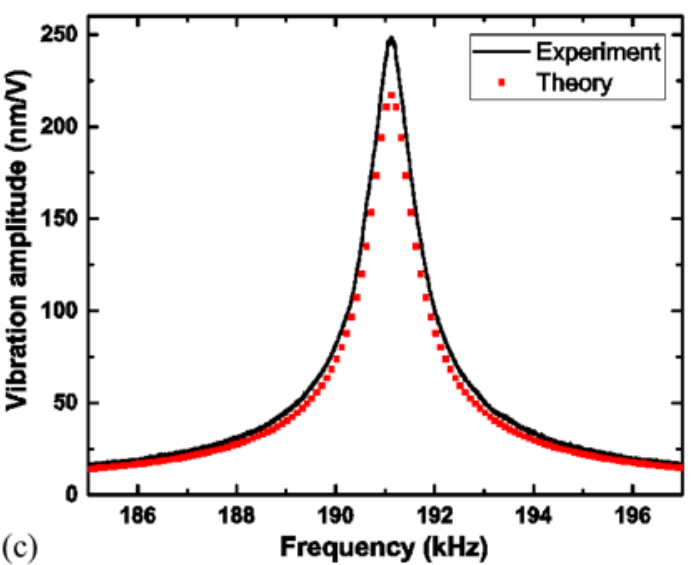

(c)

Fig. 3 a Optical image after process of a GaN beam resonator with dimensions $400 \mu \mathrm{m} \times 40 \mu \mathrm{m} \times 1.8 \mu \mathrm{m}$. The out-of-plane flexural modes are excited by the piezoelectric effect inside the Schottky diode at left. At right a resonant high-electron-mobility transistor provides amplified 
mechanical detection. b Schematic structure of the resonator in side view and cross-sectional view. c Doppler vibrometry measurement of the first mode and analytical calculation for the piezoelectric coefficient value $0.4 \mathrm{C} / \mathrm{m}^{2}$

The actuation method uses an electric field applied in the AlGaN layer of the beam from the anchor to the end of the actuation electrode. It gives a vibration amplitude that can be calculated using the model developed by DeVoe (DeVoe 2001), if there is no stress. Due to the piezoelectric property of the AlGaN, the high electric field is locally converted into an axial strain located at the top of the beam. This creates a moment which sets the beam in motion depending on its frequency response and mode shape. However, due to the tensile stress, the mode shape functions are modified, and it can be shown that at the resonant frequency the vibration amplitude is proportional to the first derivative of the corresponding mode shape function at the end of the actuation electrode according to the following formula (Ben Amar et al. 2012):

$$
\frac{u\left(x_{r}\right)}{V}=-e_{31 A l G a N} \frac{1}{2} \frac{w_{a c t}}{w} \frac{Q_{n}}{\rho 4 \pi^{2} f_{n}^{2}} \frac{U_{n}\left(x_{r}\right)}{\int_{0}^{L} U_{n}^{2}(x) d x} \frac{\partial U_{n}\left(L_{a c t}\right)}{\partial x}
$$

Here, $u\left(x_{\mathrm{r}}\right)$ is the amplitude vibration at the position $x_{\mathrm{r}}, V$ is the applied voltage amplitude, $f_{n}$ the resonant frequency, $e_{31 \mathrm{AlGaN}}$ is the effective piezoelectric coefficient, $w$ is the width of the beam, $w_{\text {act }}$ and $L$ act are the width and the length of the actuator electrode respectively and $Q_{n}$ is the quality factor. It can be noted that the integral is proportional to the length of the beam, and therefore the derivative is inversely proportional to the length of the beam. Increasing the tensile stress brings closer to the beam anchor the maximum of the first derivative of the function (Fig. 2b). Then, for beams with tensile stress, the actuation electrode has to be shorter to provide an optimal excitation.

The vibration amplitudes of the GaN beams for different modes have been measured using a laser Doppler vibrometer under atmospheric pressure and ambient temperature (Fig. 3c). For the first mode, at $191 \mathrm{kHz}$, the amplitude vibration at the center of the beam is about $250 \mathrm{~nm} / \mathrm{V}$ with a quality factor of 240 . Due to the high tensile stress, the length of the fabricated electrode is about the same as the optimal one. Without tensile stress, the optimal length of the electrode would be 0.224 times the beam length (Imboden and Mohanty 2014). In our case, the optimal length is only 0.16 times the beam length, and using a usual longer actuator would have decreased the actuator efficiency by about ten percent. Taking this into account, we found that the vibration amplitude is in good agreement with the model of the beam with tensile stress (Fig. 3c), even for higher frequency modes. However, there is a great uncertainty on the value of the piezoelectric coefficient. The previous calculations are done using $e_{31 \mathrm{AlGaN}}=0.4 \mathrm{C} / \mathrm{m}^{2}, \mathrm{but}$ theoretical or experimental values can vary with more than a factor 2 (Cimalla et al. 2007). Moreover, as the AlGaN film is thin over the $\mathrm{GaN}$, the $\mathrm{AlGaN}$ can be considered as stressed in axial and lateral direction but unstressed in the vertical direction. In these conditions, the effective piezoelectric coefficient is:

$e_{31, e f}=e_{31}-\frac{c_{13}}{c_{33}} e_{33}$

where $e_{i j}$ are the piezoelectric coefficients and $c_{i j}$ are the stiffness coefficients under constant electric field. It would be higher by a factor 1.62 , which is equal to $0.65 \mathrm{C} / \mathrm{m}^{2}$. It could also be considered that the $\mathrm{AlGaN}$ is not stressed in the lateral direction, because the $\mathrm{AlGaN}$ and $\mathrm{GaN}$ could be relaxed by a lateral deformation when an electric field is applied in the AlGaN. In that case, the effective piezoelectric coefficient is:

$e_{31, e f}{ }^{\prime}=\frac{1-{ }^{c_{12}} / c_{11}}{1-{ }^{c_{13}{ }^{2}} / c_{11} c_{33}}\left(e_{31}-\frac{c_{13}}{c_{33}} e_{33}\right)$

It would be only higher by a factor 1.08 , and equal to $0.43 \mathrm{C} / \mathrm{m}^{2}$. These higher effective piezoelectric coefficients may explain why the experimental amplitude vibration is higher than the theory shown in Fig. 3c.

Simulation with finite element method (FEM) were also performed and compared to the analytical model. The actuation efficiencies are reported as the function of the actuation electrode length for the three different effective piezoelectric coefficients and fro FEM simulation in Fig. 4. The FEM simulation are performed using anchoring boundary conditions that are fixed away from the beam on the silicon substrate so that the GaN bulk can be deformed. As expected, the FEM simulation result is between the analytical models for the thin film, and the laterally unstrained film. The FEM simulation confirms that the optimal length of the actuator is shorter than the $0.224 \times \mathrm{L}$ obtained for beam without axial stress. The lower experimental value (also shown in Fig. 4 ) could come from additional defects in the AlGaN layer or imperfect Schottky contact. 


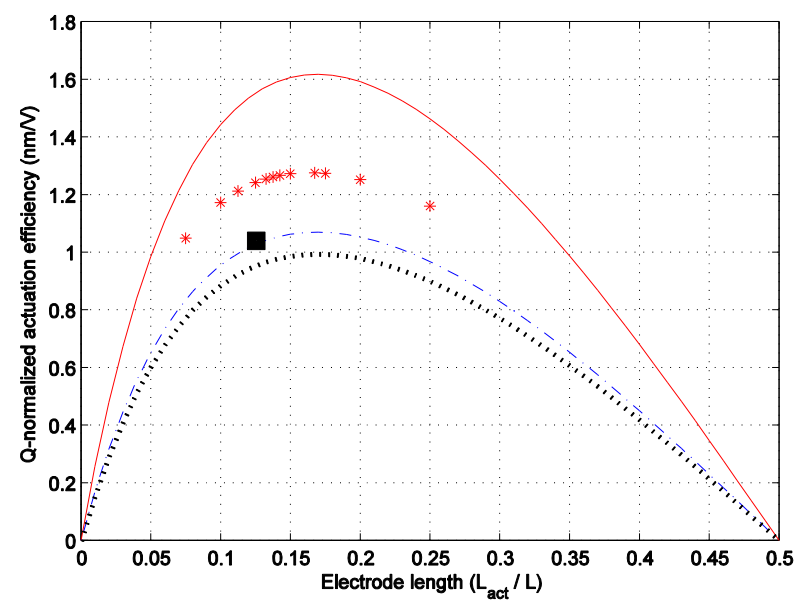

Fig. 4 Comparison between the calculated and measured actuation efficiency represented as the vibration amplitude at the center of the beam for the first mode divided by the actuation voltage and by the quality factor. The beam dimensions are $400 \mu \mathrm{m} \times 40 \mu \mathrm{m} \times 1.8 \mu \mathrm{m}$ and the internal tensile stress is $100 \mathrm{MPa}$. The red solid, blue dashed and black dotted lines are calculated form analytical models with piezoelectric coefficients equal to $0.65,0.43$ and $0.4 \mathrm{C} / \mathrm{m}^{2}$ respectively. The red stars come from FEM simulations and the black square represents the experimental value

\section{Impact of tensile stress on the quality factor}

The use of beams with high tensile stress, made possible by the MOCVD growth process, leads to an optimal design with less metal deposited on the beam compared to an unstressed beam. It minimizes the impact of the metal on the beam behavior. Indeed, the metallic electrodes have often low Young's modulus which modifies the resonant frequency, and is another source of dissipation that decreases the performances of the vibrating beam (Imboden and Mohanty 2014). Moreover, the energy stored by the vibrating beam is equal to the maximum of its kinetic energy during the motion, which is proportional to the square of the frequency (equation (1)). The energy dissipated per cycle is written $\Delta E$. Then the quality factor is:

$Q_{n}(\sigma)=2 \pi \frac{E_{c}(\sigma)}{\Delta E(\sigma)}=\frac{2 \pi}{\Delta E(\sigma)}\left((B(\sigma))^{2} \frac{\rho A}{2}\left(2 \pi f_{n}(\sigma)\right)^{2} \int_{0}^{L} U_{n}(\sigma)^{2} d x\right)$

For comparing a beam with tensile stress or without tensile stress, we scale the amplitude of vibration of the beam such that:

$(B(\sigma))^{2} \int_{0}^{L} U_{n}(\sigma)^{2} d x=(B(0))^{2} \int_{0}^{L} U_{n}(0)^{2} d x$

In other words we assume that $\frac{E_{c}(\sigma)}{E_{c}(0)}=\frac{f_{n}^{2}(\sigma)}{f_{n}^{2}(0)}$. Then we can write, using the index 0 when there is no tensile stress:

$Q_{n}(\sigma)=Q_{n, 0}\left(\frac{\Delta E_{0}}{\Delta E(\sigma)}\right)\left(\frac{f_{n}(\sigma)}{f_{n, 0}}\right)^{2}$

Then, if the dissipation mechanism is independent of the stress and the frequency, the quality factor will increase proportionally to the square of the frequency. This gives another vision than previous work about dissipation in pre-stressed beams (Schmid et al. 2011; Unterreithmeier et al. 2010) by expressing the link between the energy of the system and its resonant frequency, which can be measured experimentally.

This equation can be used for any dissipation mechanism but one has to be careful while using existing models of dissipation. For what is known as extrinsic dissipation, the models are based on the exchange of energy using the total energy of the system so that the models give directly the quality factor $Q_{n}(\sigma)$. In that case, one has to use existing models and change the value of the resonant frequency with pre-stress. Note that the presence of the resonant frequency in these models may not be explicit because it may have been replaced by its expression using the equation (4) in the stressless case. For air damping, the quality factor for presressed beams can be calculated directly by using the frequency with tensile stress in existing models (Aoust et al. 2015; Bao and Yang 2007; Blom et al. 1992; Kokubun et al. 1984). For clamping losses, the ratio of velocity of acoustic waves to the resonant frequency is decreased by the stress. So the quality factor should increase due to the tensile stress (Cross and Lifshitz 2001) by a factor going from two to three depending on the resonant mode for high tensile stress. 
For intrinsic dissipation, such as thermoelasticity, or surface or mechanical defects, the models often come from the exchange of energy only due to the flexion. So, $Q_{n, 0}\left(\Delta E_{0} / \Delta E(\sigma)\right)$ is equal to the quality factor using those models for the new resonant frequency, and the quality factors $Q_{n}(\sigma)$ associated with those dissipative mechanisms are mainly increased with tensile stress due to the increase of the frequency squared. For dissipation due to multiple materials, the impact of each material should be proportional to its energy. For example, if a metal is deposited on a beam with tensile stress, the metal should not have tensile stress so that its energy will not increase. Hence the quality factor will be higher compared to the case where the same metal is deposited on a similar beam without tensile stress.

Measurements under vacuum with a pressure of $0.1 \mathrm{mbar}$, are presented in Fig. 5, for beams with dimensions close to the previous one (see legend) and higher tensile stress. These measurements are done using the R-HEMT as electromechanical detection transducer. It confirms the increase of quality factor due to the tensile stress, with a quality factor that can be higher than 10000 which is among the highest quality factors ever reported for GaN beam resonators (Rais-Zadeh et al. 2014). Modes 2 and 3 are in good agreement with a quality factor without tensile stress $Q_{n, 0}$ that would be equal to about 1300 , and with $\Delta E_{0}=\Delta E(\sigma)$ which means that the resonant frequency and the tensile stress does not modify the dissipated energy per cycle but only increases the energy stored in the beam. For the first mode the quality factor should be higher but there is probably a limitation due to clamping losses. The constant quality factor equal to 1300 without tensile stress is attributed to surface losses. The quality factor decreases with the order of the resonant mode due to the decrease of $\gamma_{n}^{\prime}$, because the tensile stress is less dominant over the behavior of the beam for the higher mode orders.

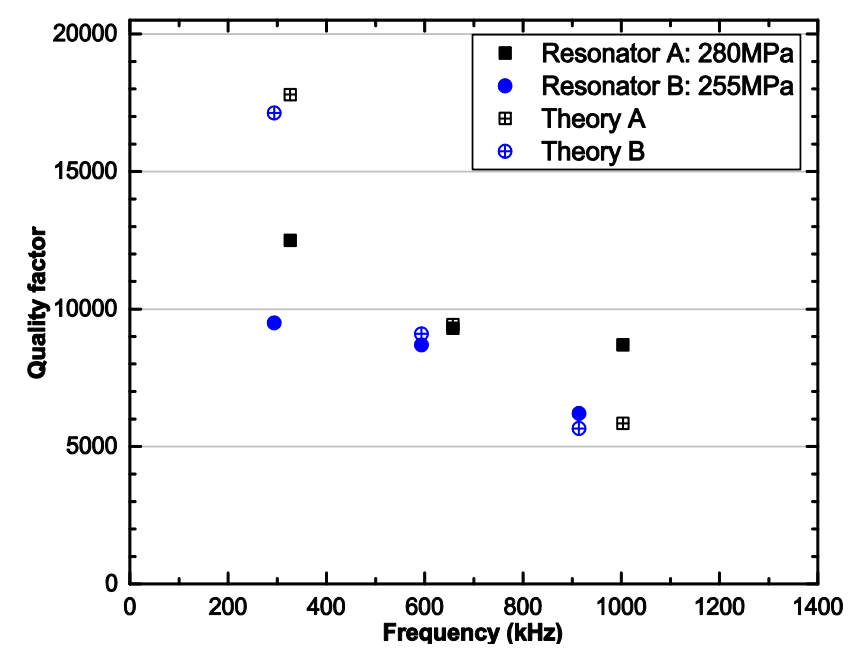

Fig. 5 Quality factor measured under vacuum for two resonators with dimensions of $380 \mu \mathrm{m} \times 20 \mu \mathrm{m} \times 1.8 \mu \mathrm{m}(\mathrm{A})$ and $390 \mu \mathrm{m} \times 20 \mu \mathrm{m} \times 1.8 \mu \mathrm{m}$ (B). The first three modes are presented showing the quality factor increase with internal tensile stress. The quality factors are obtained by fitting the resonance amplitude curve, with an uncertainty of about $10 \%$. The theory (equation 10) using $Q_{n, 0}=1300$ is plotted with crosshair symbols

\section{Conclusion}

GaN MEMS resonators with internal pre-stress were theoretically and experimentally investigated. The Young's modulus in the presence of tensile stress is mastered. The influence of the pre-stress on the resonant frequency and the mode shape functions is described. Then its effect on the actuation efficiency is analyzed showing that an optimal design requires a shorter electrode on the beam. FEM simulation and analytical models were compared to quantitatively calculate the actuation efficiency. Additionally, the tensile stress increases the stored potential energy, which might result in an increase of the quality factor. This phenomenon was confirmed by our measurements under vacuum. Therefore, since the stress of commercial epitaxial wafers is known, the design can be easily optimized and the resonant frequencies of the resonators can be determined before fabrication and test.

\section{References}

Ansari A, Gokhale VJ, Thakar VA, Roberts J, Rais-Zadeh M Gallium nitride-on-silicon micromechanical overtone resonators and filters. In: Electron Devices Meeting (IEDM), 2011 IEEE International, 2011. IEEE, pp 20.23. 21-20.23. 24

Aoust G, Levy R, Bourgeteau B, Le Traon O (2015) Viscous damping on flexural mechanical resonators Sensors and Actuators A: Physical 230:126-135 
Bao M, Yang H (2007) Squeeze film air damping in MEMS Sensors and Actuators A: Physical 136:3-27 doi:10.1016/j.sna.2007.01.008

Ben Amar A et al. (2012) Bias Dependence of Gallium Nitride Micro-Electro-Mechanical Systems Actuation Using a TwoDimensional Electron Gas Applied Physics Express 5:067201 doi:10.1143/apex.5.067201

Blom F, Bouwstra S, Elwenspoek M, Fluitman J (1992) Dependence of the quality factor of micromachined silicon beam resonators on pressure and geometry Journal of Vacuum Science \& Technology B 10:19-26

Brueckner K, Cimalla V, Niebelschütz F, Stephan R, Tonisch K, Ambacher O, Hein MA (2007) Strain- and pressure-dependent $\mathrm{RF}$ response of microelectromechanical resonators for sensing applications Journal of Micromechanics and Microengineering 17:2016-2023 doi:10.1088/0960-1317/17/10/013

Cimalla V, Pezoldt J, Ambacher O (2007) Group III nitride and SiC based MEMS and NEMS: materials properties, technology and applications Journal of Physics D: Applied Physics 40:6386-6434 doi:10.1088/0022-3727/40/20/s19

Cross MC, Lifshitz R (2001) Elastic wave transmission at an abrupt junction in a thin plate with application to heat transport and vibrations in mesoscopic systems Physical Review B 64 doi:10.1103/PhysRevB.64.085324

DenBaars SP et al. (2013) Development of gallium-nitride-based light-emitting diodes (LEDs) and laser diodes for energyefficient lighting and displays Acta Materialia 61:945-951

DeVoe DL (2001) Piezoelectric thin film micromechanical beam resonators Sensors and Actuators A: Physical 88:263-272

Faucher M, Cordier Y, Werquin M, Buchaillot L, Gaquiere C, Theron D (2012) Electromechanical Transconductance Properties of a GaN MEMS Resonator With Fully Integrated HEMT Transducers Journal of Microelectromechanical Systems 21:370-378 doi:10.1109/jmems.2011.2179010

Faucher M et al. (2009) Amplified piezoelectric transduction of nanoscale motion in gallium nitride electromechanical resonators Applied Physics Letters 94:233506 doi:10.1063/1.3153504

Imboden M, Mohanty P (2014) Dissipation in nanoelectromechanical systems Physics Reports 534:89-146 doi:10.1016/j.physrep.2013.09.003

Khan MA, Bhattarai A, Kuznia J, Olson D (1993) High electron mobility transistor based on a GaN-AlxGa1- xN heterojunction Applied Physics Letters 63:1214-1215

Kokubun K, Hirata M, Murakami H, Toda Y, Ono M (1984) A bending and stretching mode crystal oscillator as a friction vacuum gauge Vacuum 34:731-735

Leclaire P, Chenot S, Buchaillot L, Cordier Y, Théron D, Faucher M (2014) AlGaN/GaN HEMTs with very thin buffer on Si (111) for nanosystems applications Semiconductor Science and Technology 29:115018 doi:10.1088/0268$1242 / 29 / 11 / 115018$

Mishra UK, Shen L, Kazior TE, Yi-Feng W (2008) GaN-Based RF Power Devices and Amplifiers Proceedings of the IEEE 96:287-305 doi:10.1109/jproc.2007.911060

Popa LC, Weinstein D Switchable piezoelectric transduction in AlGaN/GaN MEMS resonators. In: Solid-State Sensors, Actuators and Microsystems (TRANSDUCERS \& EUROSENSORS XXVII), 2013 Transducers \& Eurosensors XXVII: The 17th International Conference on, 2013. IEEE, pp 2461-2464

Rais-Zadeh M, Gokhale VJ, Ansari A, Faucher M, Théron D, Cordier Y, Buchaillot L (2014) Gallium nitride as an electromechanical material Microelectromechanical Systems, Journal of 23:1252-1271

Schmid S, Jensen KD, Nielsen KH, Boisen A (2011) Damping mechanisms in high-Qmicro and nanomechanical string resonators Physical Review B 84 doi:10.1103/PhysRevB.84.165307

Torri G et al. (2014) Piezoelectric transduction of flexural modes in pre-stressed microbeam resonators Journal of Micromechanics and Microengineering 24:085014

Unterreithmeier QP, Faust T, Kotthaus JP (2010) Damping of nanomechanical resonators Phys Rev Lett 105:027205 doi:10.1103/PhysRevLett.105.027205 